\title{
Low-dose aspirin might alleviate the symptoms of preeclampsia by increasing the expression of antioxidative enzymes
}

\author{
WEN XIONG ${ }^{1}$, YANJUN WANG ${ }^{2}$ and XINE ZHOU $^{1}$ \\ ${ }^{1}$ Department of Obstetrics, Chengdu Women's and Children's Central Hospital, School of \\ Medicine, University of Electronic Science and Technology of China; ${ }^{2}$ Department of Geriatrics, \\ West China Hospital of Sichuan University, Chengdu, Sichuan 610000, P.R. China
}

Received December 14, 2019; Accepted June 22, 2021

DOI: $10.3892 /$ etm.2021.10853

\begin{abstract}
Preeclampsia (PE) is a pregnancy-related syndrome that is characterized by new onset of hypertension combined with proteinuria or end-organ dysfunction occurring after 20 weeks of pregnancy. Endothelial dysfunction is also commonly observed in patients with PE. PE remains a leading cause of maternal morbidity and mortality, resulting in $\sim 76,000$ maternal and 500,000 fetus and newborn deaths worldwide annually. The present study aimed to investigate the protective effect of aspirin in patients with PE. A PE model was established in $\mathrm{C} 57 / \mathrm{BL}$ mice, followed by the detection of expression levels of antioxidative enzymes, including superoxide dismutase 1 , catalase, periaxin and thioredoxin and AKT/mTOR signaling pathway-related proteins by performing western blotting. The concentration of these enzymes in serum samples from PE model mice was also assessed. Compared with the negative control group, the expression of these antioxidative enzymes was decreased in $\mathrm{PE}$ model mice $(\mathrm{P}<0.05)$. High-dose aspirin treatment enhanced PE-induced effects, whereas low-dose aspirin treatment partially reversed PE-induced effects $(\mathrm{P}<0.05)$. Moreover, the results indicated that the effects of aspirin treatment on PE might be mediated via the AKT/mTOR signaling pathway. Therefore, low-dose aspirin administration may serve as a therapeutic strategy for PE.
\end{abstract}

\section{Introduction}

Preeclampsia (PE) is defined as the occurrence of new onset hypertension and proteinuria or other target organ damage occurring after 20 weeks of gestation (1). PE affects $\sim 5 \%$

Correspondence to: Dr Wen Xiong, Department of Obstetrics, Chengdu Women's and Children's Central Hospital, School of Medicine, University of Electronic Science and Technology of China, 1617 Riyue Avenue, Qingyang, Chengdu, Sichuan 610000, P.R. China

E-mail: wen_xiong2018@outlook.com

Key words: aspirin, preeclampsia, oxidative stress, AKT/mTOR signaling pathway of pregnancies worldwide and is one of the most commonly observed complications during pregnancy (2). The disease not only causes maternal and fetal morbidity and mortality, but also leads to premature birth of the fetus and long-term cardiovascular disease in pregnant women (3). In 1914, researchers found that pregnant women with toxemia, albuminuria and eclampsia presented with an increased incidence rate of PE compared with women with healthy pregnancies (4), which might be mediated by placental hypoperfusion and ischemia. The development of PE is a complex process, including abnormal homeostasis of placental cells, oxidative stress response and angiogenesis (5). Abnormal oxidative stress leads to abnormal spiral artery remodeling in the placenta, further impairing angiogenesis process (6), which also contributes to the pathophysiology of PE (7). Altering the angiogenesis process could induce hypoperfusion and ischemia in the placenta, resulting in placental trophoblastic cell apoptosis, which is critical for maintaining placental homeostasis $(8,9)$, which leads to the occurrence of PE. Aspirin, a pharmaceutical drug, affects the inflammatory cascade by irreversibly inhibiting the activity of cyclooxygenase (COX)-1 and COX-2, further inhibiting the production of prostaglandins and thromboxane (10). Inhibition of COX activity can result in inhibition of thromboxane A2, a decrease in the release of cytokines, growth factors and coagulation factors (11), accumulation of reactive oxygen species (ROS) and inhibition of the oxidative stress response process (12). A previous study reported that pregnant women administered low-dose aspirin treatment (60-150 mg/day) displayed a significant reduction in the occurrence of preterm birth and PE frequency (13). Another study demonstrated that 100-160 mg aspirin treatment decreased the risk of PE starting from 16 weeks of gestation compared with patients receiving $300 \mathrm{mg}$ aspirin treatment (14). Therefore, we hypothesize that aspirin may serve as a potential therapeutic strategy for PE. The present study established a PE model in C57/BL mice and evaluated the change in expression levels of antioxidative enzymes and the AKT/mTOR signaling pathway to elucidate the effects and mechanism of aspirin treatment on PE.

\section{Materials and methods}

Materials. A total protein extraction kit (cat. no. BC3711) and BCA protein assay kit (cat. no. PC0020) were purchased from 
Beijing Solarbio Science \& Technology Co., Ltd. Primary antibodies targeted against CAT (cat. no. ab16731), SOD1 (cat. no. ab13498), TRX (cat. no. ab26320) and periaxin (PRX; cat. no. ab211292) were purchased from Abcam. A total SOD assay kit (cat. no. A001-1-2) and TRX peroxidase test kit were purchased from Nanjing Jiancheng Bioengineering Institute. A human TRX ELISA kit (cat. no. CSB-E09728h) and human CAT ELISA kit (cat. no. CSB-E13635h) were purchased from Cusabio Technology LLC.

Ethics statements. The present study was approved by the Ethics Committee of Chengdu Women's and Children's Central Hospital (approval no. SCXK 2009-0017; Chengdu, China). The treatment of all 80 animals complied with the Guide for the Care and Use of Laboratory Animals (15).

Animal treatment and grouping. A total of 80 female C57/BL mice (weight, 20-22 g; age, 7 weeks) and 80 male mice (weight, 21-25 g; age, 8 weeks) were purchased from the Laboratory Animal Center of the Academy of Military Medical Sciences of Beijing People's Liberation Army (Beijing, China). Female and male mice were housed at $24^{\circ} \mathrm{C}$ with $60-70 \%$ humidity, 12-h dark/light cycles and free access to food and water. Female mice were mated with male mice overnight during the proestrus period and timed for pregnancy after 1 week of adaptation. Mice were divided into four groups ( $n=20 /$ group) as follows: i) Negative control group (NC); ii) PE group (PE); iii) PE with low-dose aspirin ( $2 \mathrm{mg} / \mathrm{kg}$ ) treatment group (AL); and iv) $\mathrm{PE}$ with high-dose $(10 \mathrm{mg} / \mathrm{kg})$ aspirin treatment group (AH). Mice in the PE model group were subcutaneously injected with L-N ${ }^{\mathrm{G}}$-Nitroarginine methyl ester (L-NAME; $50 \mathrm{mg}$ /day; Sigma-Aldrich; Merck KGaA) at gestation day (GD) 7 until GD18. The concentration of proteinuria was measured at GD7 and GD18 by performing a BCA assay. Blood pressure was measured at GD 7, 10, 13, 16 and 18 using an animal non-invasive sphygmomanometer (cat. no. BP-98A; Softron Co., Ltd.). Mice in the NC group were treated with equal volume of normal saline.

Tissue collection and processing. After L-NAME treatment at GD 18, mice were anesthetized with isoflurane (3\% for induction, $1.5 \%$ for maintenance; Sigma-Aldrich; Merck KGaA) in an induction chamber. Urine samples were collected from the anesthetized mice, and then blood samples $(0.5 \mathrm{ml})$ were collected by removing the eyeballs. The blood samples were stored at $4^{\circ} \mathrm{C}$ until further processing. The mice were then sacrificed by cervical dislocation and the placental tissues were collected by full-term caesarian section. The obtained placental tissues were transferred in a petri dish, washed with sterile normal saline to remove the blood and stored in liquid nitrogen until further analysis. The obtained blood samples were processed within $4 \mathrm{~h}$ of collection. Briefly, the blood samples were centrifuged at $1,006 \mathrm{x}$ g for $15 \mathrm{~min}$ at $4^{\circ} \mathrm{C}$ to obtain serum, which was stored at $-80^{\circ} \mathrm{C}$ until further analysis.

Protein extraction and western blotting. Total protein was extracted from placental tissue using the total protein extraction kit (cat. no. 2140; Millipore Sigma) according to the manufacturer's protocol. Briefly, placental tissues were homogenized with lysis buffer (Thermo Fisher Scientific, Inc.) using a homogenizer, and the supernatant was collected after centrifugation $(16,099 \mathrm{x} \mathrm{g})$ at $4^{\circ} \mathrm{C}$ for $10 \mathrm{~min}$. Protein concentrations were determined using the BCA assay. Subsequently, proteins $(60 \mu \mathrm{g})$ were separated via 10\% SDS-PAGE and transferred onto $0.22 \mu \mathrm{m}$ nitrocellulose membranes (Millipore Sigma) using a semi-dry blotter. Following blocking with 5\% non-fat milk for $1 \mathrm{~h}$ at room temperature, the membranes were incubated with primary antibodies (all Cell Signaling Technology, Inc.) against GAPDH (1:5,000; cat. no. 5174), p-AKT (1:1,000; cat. no. 9271), AKT (1:1,000; cat. no. 9272), p-mTOR (1:1,000; cat. no. 2971), mTOR $(1: 1,000$; cat. no. 2972) and PTEN (1:1,000; cat. no. 9188) at $4^{\circ} \mathrm{C}$ overnight. Subsequently, the membranes were incubated with HRP-conjugated goat anti-mouse IgG secondary antibodies (1:1,000; cat. no. PV-6001; OriGene Technologies, Inc.) for $1 \mathrm{~h}$ at room temperature. Protein bands were visualized using chemiluminescence (Amersham; Cytiva). Protein expression levels were semi-quantified using Image-Pro Plus software (version 6.0; Media Cybernetics, Inc.).

ELISA. ELISAs were performed using ELISA kits according to the manufacturer's protocols. Briefly, Serum samples were added into each well of a 96-plate. After incubation with the reaction buffer at $37^{\circ} \mathrm{C}$, the optical density value of each well was measured at a wavelength of $450 \mathrm{~nm}$ using a microplate reader.

Statistical analysis. Data are presented as the mean \pm SD. Each experiment was repeated three times independently. Dunnett's post hoc test was used for comparisons among multiple groups following one-way ANOVA using SPSS software version 19.0 (IBM Corp.). $\mathrm{P}<0.05$ was considered to indicate a statistically significant difference.

\section{Results}

Measurement of blood pressure of mice in each group. The blood pressure of mice in each group are presented in Fig. 1A. Compared with the NC group, the blood pressure was not altered at GD7 but notably increased in all treatment groups at GD10. The blood pressure in the PE group remained stable at GD13, 16 and 18; however, blood pressure displayed a different trend in the $\mathrm{AH}$ and $\mathrm{AL}$ groups. Blood pressure in the AH group gradually increased to $\sim 175 \mathrm{mmHg}$ by GD18, whereas blood pressure in the AL group gradually decreased to $\sim 120 \mathrm{mmHg}$ by GD18.

Concentration of proteinuria in each group. As shown in Fig. 1B, the concentration of proteinuria at GD7 was $0.31 \pm 0.04$, $0.35 \pm 0.06,0.29 \pm 0.05$ and $0.32 \pm 0.03$ in the NC, PE, AH and AL groups, respectively. At GD18, the concentration of proteinuria was $0.25 \pm 0.03,0.92 \pm 0.08,1.82 \pm 0.15$ and $0.31 \pm 0.04$ in the $\mathrm{NC}$, $\mathrm{PE}, \mathrm{AH}$ and AL groups, respectively (Fig. 1C). The concentration of proteinuria was significantly increased in $\mathrm{PE}$ and $\mathrm{AH}$ groups compared with the NC group at GD18 $(\mathrm{P}<0.05)$.

Expression levels of AKT/mTOR signaling pathway-related proteins in placental tissues. As shown in Fig. 2A and B, the ratios of $\mathrm{p}-\mathrm{AKT} / \mathrm{AKT}$ was $0.57 \pm 0.05,0.61 \pm 0.05,0.49 \pm 0.04$ and $1.09 \pm 0.09$ in the NC, PE, AH and AL groups, respectively. The ratio of $\mathrm{p}-\mathrm{AKT} / \mathrm{AKT}$ was significantly increased in the $\mathrm{AH}$ 

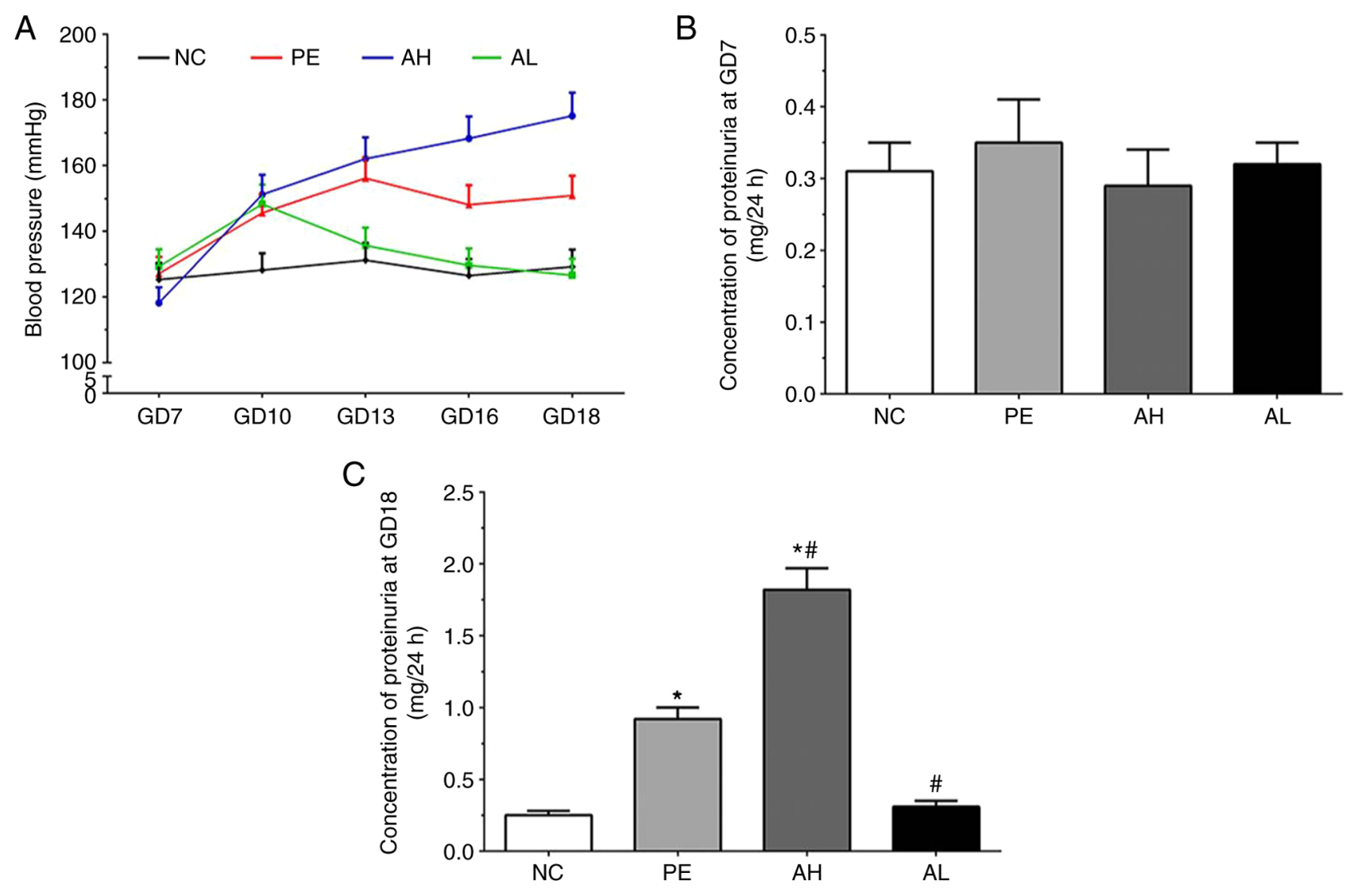

Figure 1. Evaluation of PE mouse model. (A) Changes in blood pressure. Concentration of proteinuria in mice at (B) GD7 (without L-NAME treatment) and (C) 18 (after L-NAME treatment). Data are presented as the mean $\pm \mathrm{SD}$. ${ }^{*} \mathrm{P}<0.05$ vs. NC; ${ }^{*} \mathrm{P}<0.05$ vs. PE. PE, preeclampsia; GD, gestation day; L-NAME, L-N ${ }^{\mathrm{G}}$-Nitroarginine methyl ester; NC, negative control; AH, PE with high-dose $(10 \mathrm{mg} / \mathrm{kg})$ aspirin treatment; AL, PE with low-dose aspirin $(2 \mathrm{mg} / \mathrm{kg})$ treatment.

group $(\mathrm{P}<0.05)$ compared with the NC group, but significantly decreased in the AL group $(\mathrm{P}<0.05)$ and significantly increased in the AH group $(\mathrm{P}<0.05)$ compared with the $\mathrm{PE}$ group. The ratios of $\mathrm{p}$-mTOR/mTOR were $1.23 \pm 0.10,1.17 \pm 0.10,0.74 \pm 0.06$ and $1.22 \pm 0.10$ in the $\mathrm{NC}, \mathrm{PE}, \mathrm{AH}$ and $\mathrm{AL}$ groups, respectively (Fig. 2A and C). The ratio of $\mathrm{p}-\mathrm{mTOR} / \mathrm{mTOR}$ was significantly decreased in the AL group $(\mathrm{P}<0.05)$ compared with the NC and PE groups. The expression levels of PTEN were $0.95 \pm 0.08,1.53 \pm 0.13,1.63 \pm 0.14$ and $0.71 \pm 0.06$ in the NC, PE, AH and AL groups, respectively (Fig. 2A and D). Compared with the NC group, the expression of PTEN was significantly increased in the $\mathrm{PE}$ and $\mathrm{AL}$ groups $(\mathrm{P}<0.05)$, but significantly decreased in the $\mathrm{AH}$ group $(\mathrm{P}<0.05)$. The expression of PTEN was also significantly decreased in the AH group $(\mathrm{P}<0.05)$ compared with the PE group.

Expression of antioxidative proteins in placental tissues. As shown in Fig. 3, the expression levels of CAT, SOD1, TRX and PRX were detected by performing western blotting. The expression levels of CAT in the NC, $\mathrm{PE}, \mathrm{AH}$ and AL groups were $1.06 \pm 0.09,0.71 \pm 0.06,0.55 \pm 0.05$ and $0.89 \pm 0.07$, respectively. The expression of CAT was significantly decreased in the $\mathrm{PE}$ and $\mathrm{AH}$ groups $(\mathrm{P}<0.05)$ compared with the $\mathrm{NC}$ group. Compared with the PE group, the expression of CAT was significantly decreased in the AH group $(\mathrm{P}<0.05)$, but significantly increased in the $\mathrm{AL}$ group $(\mathrm{P}<0.05)$. The expression levels of PRX in the NC, PE, AH and AL groups were $0.83 \pm 0.07$,
$0.74 \pm 0.06,0.65 \pm 0.05$ and $0.90 \pm 0.07$, respectively. Compared with the NC group, the expression of PRX was significantly decreased in the AH group $(\mathrm{P}<0.05)$. Compared with the PE group, the expression of PRX was significantly increased in the AL group $(\mathrm{P}<0.05)$. The expression levels of TRX were $0.90 \pm 0.08,0.81 \pm 0.07,0.66 \pm 0.06$ and $0.95 \pm 0.08$ in the NC, PE, $\mathrm{AH}$ and $\mathrm{AL}$ groups, respectively. The expression of TRX was significantly decreased in the AH group $(\mathrm{P}<0.05)$ compared with the NC and PE groups, but significantly increased in the AL group $(\mathrm{P}<0.05)$ compared with the $\mathrm{PE}$ group. The expression levels of SOD1 were $1.03 \pm 0.09,0.81 \pm 0.07,0.80 \pm 0.07$ and $0.98 \pm 0.08$ in the NC, PE, AH and AL groups, respectively. The expression of SOD1 was significantly decreased in the $\mathrm{PE}$ and $\mathrm{AH}$ groups $(\mathrm{P}<0.05)$ compared with the $\mathrm{NC}$ group. By contrast, compared with the PE group, the expression of SOD1 was significantly increased in the AL group $(\mathrm{P}<0.05)$.

Measurement of oxidative enzymes in serum samples of mice. As shown in Fig. 4A, the concentrations of SOD in serum samples of the $\mathrm{NC}, \mathrm{PE}, \mathrm{AH}$ and $\mathrm{AL}$ groups were 2.18 \pm 0.21 , $1.62 \pm 0.15,1.53 \pm 0.17$ and $2.34 \pm 0.19 \mathrm{ng} / \mathrm{ml}$, respectively. The concentration of SOD was significantly decreased in the PE and AH groups $(\mathrm{P}<0.05)$ compared with the $\mathrm{NC}$ group, but significantly increased in the AL group $(\mathrm{P}<0.05)$ compared with the PE group. The concentrations of PRX in serum samples of the NC, PE, AH and AL groups were $65.26 \pm 2.31,47.31 \pm 1.98$, $40.51 \pm 1.62$ and $73.83 \pm 3.05 \mathrm{ng} / \mathrm{ml}$, respectively. Compared 

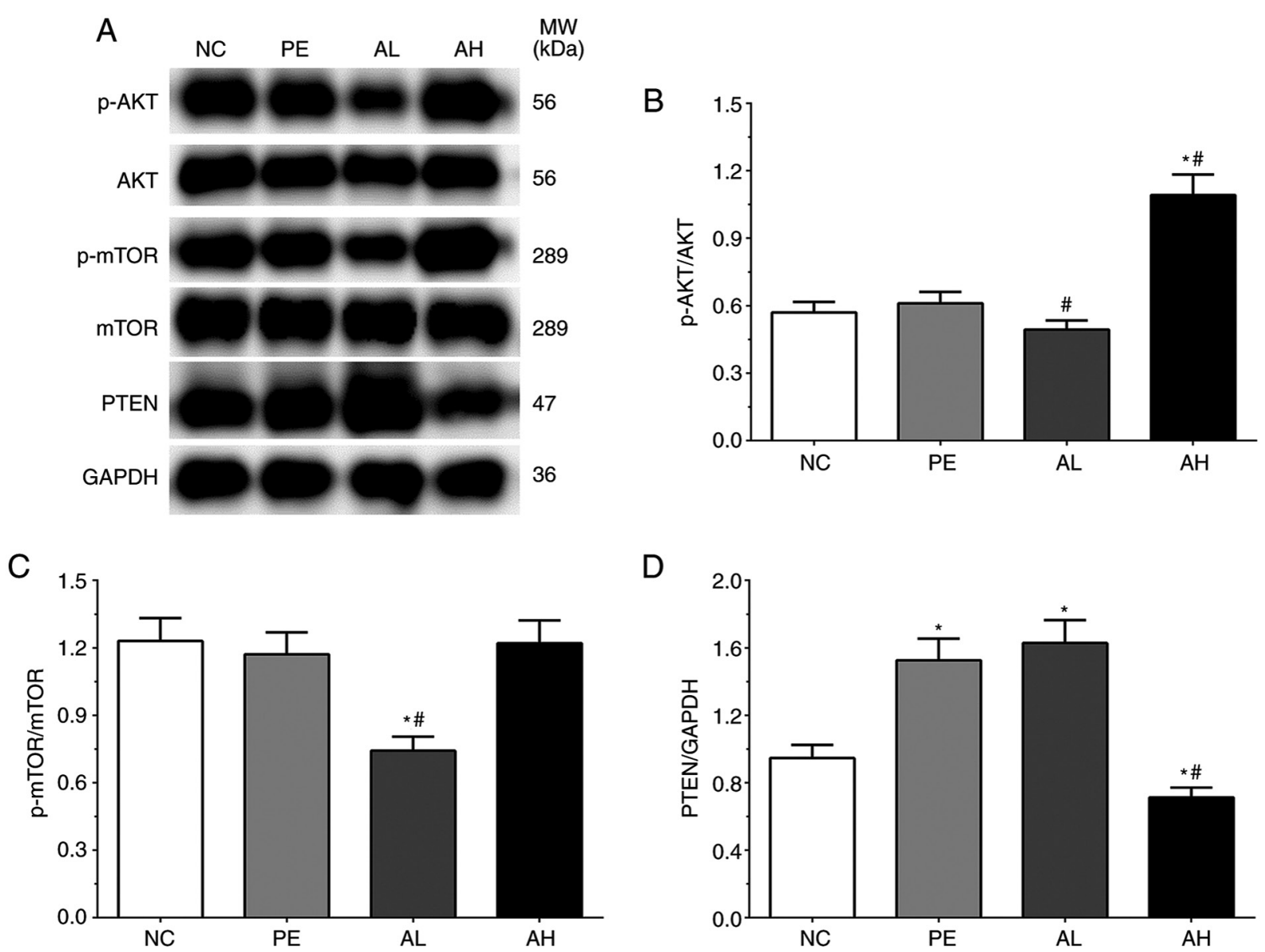

Figure 2. AKT/mTOR signaling pathway-related protein expression levels in placental tissue of PE model mice. Protein expression levels were (A) determined by western blotting and semi-quantified for (B) p-AKT/AKT, (C) p-mTOR/mTOR and (D) PTEN. Each experiment was repeated three times independently. Data are presented as the mean $\pm \mathrm{SD}$. ${ }^{*} \mathrm{P}<0.05$ vs. $\mathrm{NC} ;{ }^{*} \mathrm{P}<0.05$ vs. PE. PE, preeclampsia; $\mathrm{p}$, phosphorylated; $\mathrm{NC}$, negative control; AL, $\mathrm{PE}$ with low-dose aspirin $(2 \mathrm{mg} / \mathrm{kg})$ treatment; AH, PE with high-dose $(10 \mathrm{mg} / \mathrm{kg})$ aspirin treatment; $\mathrm{MW}$, molecular weight.
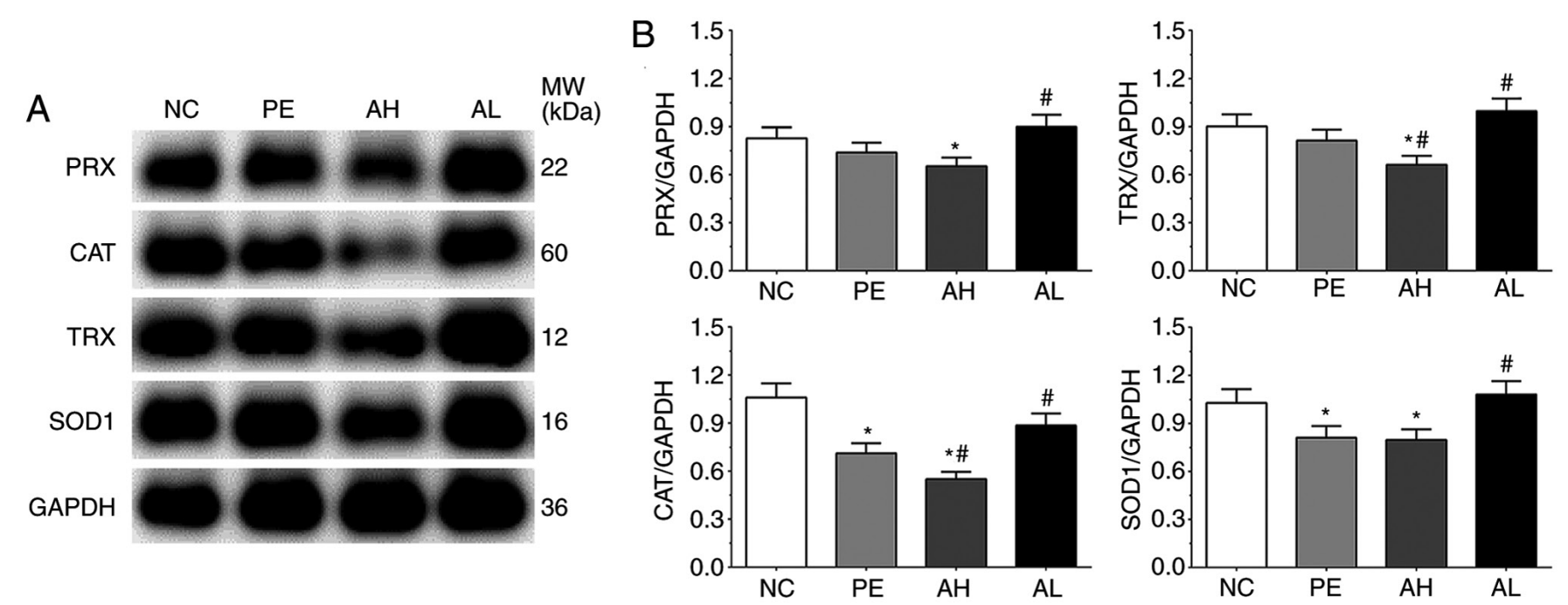

Figure 3. Antioxidative enzyme expression levels in placental tissue of PE model mice. PRX, CAT, TRX and SOD1 protein expression levels were (A) determined by western blotting and (B) semi-quantified. Each experiment was repeated three times independently. Data are presented as the mean \pm SD. ${ }^{*} \mathrm{P}<0.05$ vs. NC; ${ }^{\text {P }}<0.05$ vs. PE. PE, preeclampsia; PRX, periaxin; CAT, catalase; TRX, thioredoxin; SOD1, superoxide dismutase 1; NC, negative control; AH, PE with high-dose $(10 \mathrm{mg} / \mathrm{kg})$ aspirin treatment; AL, PE with low-dose aspirin $(2 \mathrm{mg} / \mathrm{kg})$ treatment; MW, molecular weight.

with the NC group, the concentration of PRX was significantly decreased in $\mathrm{PE}$ and $\mathrm{AH}$ groups $(\mathrm{P}<0.05)$, but significantly increased in the AL group $(\mathrm{P}<0.05)$. The concentration of PRX was also significantly decreased in the AH group $(\mathrm{P}<0.05)$ and significantly increased in the $\mathrm{AL}$ group $(\mathrm{P}<0.05)$ compared with the PE group. The concentrations of TRX in 
A

ह

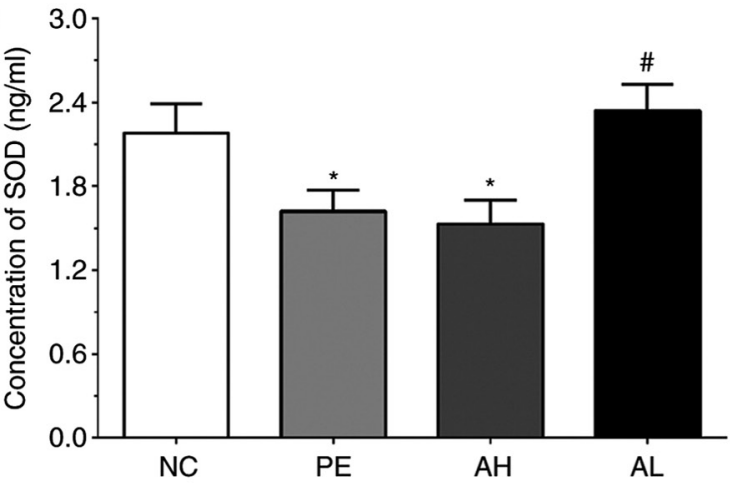

C

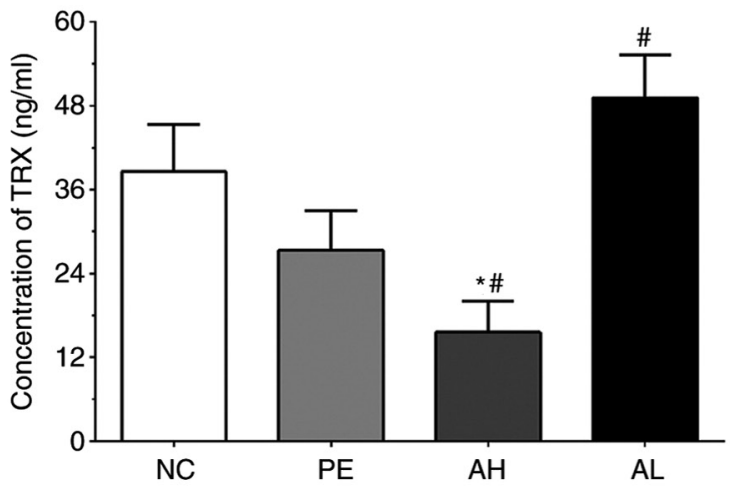

$\mathrm{B}$

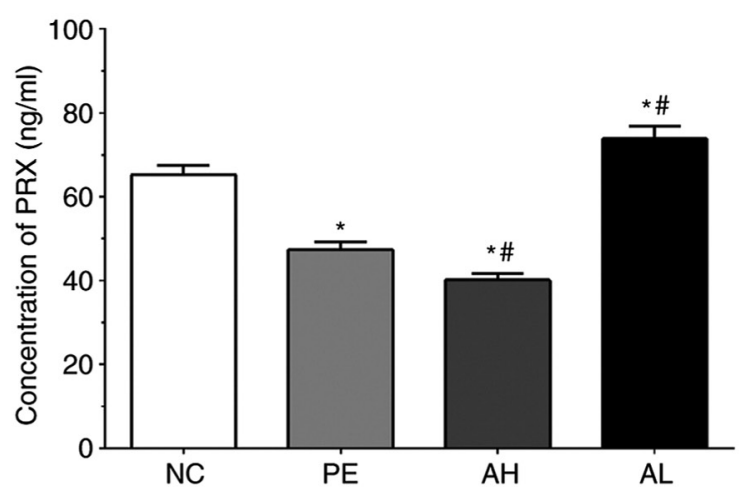

D

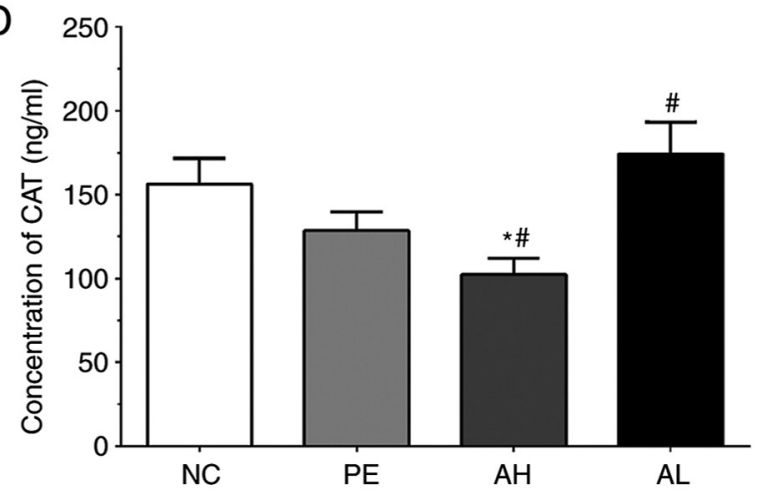

Figure 4. Concentrations of antioxidative enzymes in serum samples of PE model mice. Concentration of (A) SOD, (B) PRX, (C) TRX and (D) CAT in serum samples of PE model mice. Each experiment was repeated three times independently. Data are presented as the mean $\pm \mathrm{SD}$. ${ }^{*} \mathrm{P}<0.05$ vs. NC; ${ }^{\prime} \mathrm{P}<0.05$ vs. $\mathrm{PE}$. PE, preeclampsia; SOD, superoxide dismutase; PRX, periaxin; TRX, thioredoxin; CAT, catalase; AH, PE with high-dose (10 mg/kg) aspirin treatment; AL, PE with low-dose aspirin $(2 \mathrm{mg} / \mathrm{kg})$ treatment.

the NC, PE, AH and AL groups were 38.56 $\pm 6.82,27.31 \pm 5.65$, $15.58 \pm 4.51$ and $49.13 \pm 6.21 \mathrm{ng} / \mathrm{ml}$, respectively. The concentration of TRX was significantly decreased in the AH group $(\mathrm{P}<0.05)$ compared with the NC and PE groups, but significantly increased in the AL group $(\mathrm{P}<0.05)$ compared with the PE group. The concentrations of CAT were 156.22 \pm 15.42 , $128.43 \pm 11.23,102.31 \pm 9.81$ and $174.15 \pm 19.17 \mathrm{pg} / \mathrm{ml}$ in the $\mathrm{NC}$, $\mathrm{PE}, \mathrm{AH}$ and $\mathrm{AL}$ groups, respectively. The concentration of CAT was significantly decreased in the AH group $(\mathrm{P}<0.05)$ compared with the $\mathrm{NC}$ and $\mathrm{PE}$ groups, but significantly increased in the AL group $(\mathrm{P}<0.05)$ compared with the $\mathrm{PE}$ group.

\section{Discussion}

PE is defined as new onset hypertension and proteinuria or other damage in organs that occurs after 20 weeks of gestation (16). PE affects $~ 5 \%$ of pregnancies worldwide and remains a leading complication of pregnancy (17), causing maternal and fetal morbidity and mortality. Aspirin, an antiplatelet reagent, is widely used for the treatment of cardiovascular disease (10). A previous study found that the vascular production of prostacyclin was deficient in PE, and the increased production of thromboxane could lead to the aggregation of platelets, which is commonly observed in patients with PE (18). A previous study indicated that low-dose aspirin might be useful for protecting pregnant women against the development of PE (19). The present study established a
PE model in pregnant mice and explored the protective role of low-dose aspirin for PE. Compared with the NC group, the expression levels and concentrations of antioxidative enzymes, including CAT, TRX, PRX and SOD, in serum samples and placental tissues were decreased in the PE model, respectively. The expression levels and concentrations of these antioxidative enzymes were further decreased after high-dose aspirin treatment, whereas PE-mediated effects were significantly reversed after low-dose aspirin treatment. The results indicated that aspirin treatment-induced alterations were mediated via the AKT/mTOR signaling pathway.

Pregnancy has been reported to increase the oxidative stress status of pregnant women, resulting in an increase in circulating ROS. The major organ that regulates ROS during pregnancy is the placenta (20). The function of antioxidative stress defenses is also impaired by the downregulation of antioxidant enzymes (21). Multiple enzymes participate in the maintenance of oxidative status balance, including CAT, glutathione peroxidase and peroxiredoxins (22). Among them, CAT contains a $\mathrm{H}_{2} \mathrm{O}_{2}$ dismutase, which is localized in the peroxisome and removes the extra $\mathrm{H}_{2} \mathrm{O}_{2}$ or prevents $\mathrm{H}_{2} \mathrm{O}_{2}$ leakage (23). TRX is a protein that is characterized by a catalytically active dithiol site, and widely exists in bacteria, plants and animals (24). TRX catalyzes the cleavage of disulfide bonds of downstream proteins and converts them into the oxidized form. TRX can also quench ROS via cooperation with TRX-dependent peroxidases or PRX (25). TRX reductase can transfer electrons from NADPH to TRX 
to decrease disulfide linkage formation in PRX through peroxidase reaction (26). SOD1 is also a critical enzyme in the antioxidative system; in the cytoplasm, SOD1 dismutates the superoxide anion radical into $\mathrm{H}_{2} \mathrm{O}_{2}$, which is further reduced by CAT, glutathione peroxidases or PRX into $\mathrm{H}_{2} \mathrm{O}$ (27). SOD1 performs a similar protective role in the regulation of superoxide in the intermembrane space as in the cytosol (28). The present study demonstrated that the increased oxidative level in the PE mouse model was decreased after low-dose aspirin treatment. Hence, low-dose aspirin administration may serve as a therapeutic strategy for PE.

ROS also participates in the production of multiple genes involved in cell differentiation and proliferation, affecting the cellular defense system and mitogenic pathway (29). A low level of ROS promotes the angiogenesis process in the placenta during pregnancy via upregulation of transcription factor E26 transformation-specific oncogene homolog 1, resulting in the upregulation of VEGF. Moreover, a low level of ROS can also promote the invasion of cells via upregulation of kruppel-like factor 8 and activation of matrix metalloproteinase 9 (30). The PI3K/AKT/mTOR signaling pathway serves an important role in the regulation of cellular proliferation via multiple mechanisms. A high level of ROS can elevate proproliferative signaling with inhibition of growth suppressors via activation of the PI3K/AKT signaling pathway through inhibition of phosphatases of PTEN (31). PTEN negatively regulates the PI3K/AKT signaling pathway via dephosphorylation of phosphatidylinositol $(3,4,5)$-trisphosphate to phosphatidylinositol $(4,5)$-bisphosphate (32). PI3K/AKT further promotes cell proliferation via the mTORC1-dependent signaling pathway (33). A previous study demonstrated that AKT could directly phosphorylate mitochondrial GSK-3 $\beta$, leading to decreases in GSK-3 $\beta$ activity and alleviation of negative regulation mediated by pyruvate dehydrogenase and $\alpha$-ketoglutarate dehydrogenase complexes, which results in the generation of superoxide and $\mathrm{H}_{2} \mathrm{O}_{2}$ (34). The present study demonstrated that, compared with the PE group, activation of the AKT/mTOR signaling pathway was significantly suppressed in the low-aspirin treatment group, and AKT signaling was significantly promoted in the high-aspirin treatment group, indicating that the oxidative stress process was activated after high-dose aspirin treatment. Furthermore, compared with the PE group, the expression of PTEN was not significantly increased in the low-dose aspirin treatment group, but significantly decreased in the high-dose aspirin treatment group, also resulting in the activation of the AKT/mTOR signaling pathway.

In the present study, a model of PE in C57/BL mice was established. Subsequently, the blood pressure and proteinuria concentrations were measured to confirm successful establishment of the model. The expression levels and concentrations of antioxidative stress enzymes in the placental tissues and circulating serum samples were determined by performing western blotting and ELISAs, respectively. The results indicated that, compared with the NC group, the expression of antioxidative stress enzymes was decreased in the PE model and further decreased after high-dose aspirin treatment, but these PE-induced effects were partially reversed after low-dose aspirin treatment. Furthermore, the results suggested that aspirin-induced effects might be mediated via the
$\mathrm{PI} 3 \mathrm{~K} / \mathrm{AKT} / \mathrm{mTOR}$ signaling pathway and regulation of ROS production. Therefore, low-dose aspirin administration may serve as a therapeutic strategy for PE.

\section{Acknowledgements}

Not applicable.

\section{Funding}

No funding was received.

\section{Availability of data and materials}

The datasets used and/or analyzed during the current study are available from the corresponding author on reasonable request.

\section{Authors' contributions}

WX designed the study and drafted and revised the manuscript. YW and XZ collected, analyzed and interpreted data. WX and $\mathrm{XZ}$ confirm the authenticity of all the raw data. All authors read and approved the final manuscript.

\section{Ethics approval and consent to participate}

The present study was approved by the Ethics Committee of Chengdu Women's and Children's Central Hospital (approval no. SCXK 2009-0017; Chengdu, China).

\section{Patient consent for publication}

Not applicable.

\section{Competing interests}

The authors declare that they have no competing interests.

\section{References}

1. No authors listed: Hypertension in pregnancy. Report of the American College of Obstetricians and Gynecologists' Task Force on Hypertension in Pregnancy. Obstet Gynecol 122: 1122-1131, 2013.

2. Abalos E, Cuesta C, Grosso AL, Chou D and Say L: Global and regional estimates of preeclampsia and eclampsia: A systematic review. Eur J Obstet Gynecol Reprod Biol 170: 1-7, 2013.

3. Kuklina EV, Ayala C and Callaghan WM: Hypertensive disorders and severe obstetric morbidity in the United States. Obstet Gynecol 113: 1299-1306, 2009.

4. Young J: The AEtiology of eclampsia and albuminuria and their relation to accidental haemorrhage: (An anatomical and experimental investigation). Proc R Soc Med 7: 307-348, 1914.

5. Phipps E, Prasanna D, Brima W and Jim B: Preeclampsia: Updates in pathogenesis, definitions, and guidelines. Clin J Am Soc Nephrol 11: 1102-1113, 2016.

6. Crocker IP, Tansinda DM and Baker PN: Altered cell kinetics in cultured placental villous explants in pregnancies complicated by pre-eclampsia and intrauterine growth restriction. J Pathol 204: 11-18, 2004.

7. Pereira RD, De Long NE and Wang RC: Angiogenesis in the placenta: The role of reactive oxygen species signaling. Biomed Res Int 2015: 814543, 2015.

8. Zhang Z, Wei C, Zhou Y, Yan T, Wang Z, Li W and Zhao L: Homocysteine induces apoptosis of human umbilical vein endothelial cells via mitochondrial dysfunction and endoplasmic reticulum stress. Oxid Med Cell Longev 2017: 5736506, 2017. 
9. Wu F, Tian FJ and Lin Y: Oxidative stress in placenta: Health and diseases. Biomed Res Int 2015: 293271, 2015.

10. Vane JR and Botting RM: The mechanism of action of aspirin. Thromb Res 110: 255-258, 2013.

11. Schrottmaier WC, Kral JB, Badrnya S and Assinger A: Aspirin and P2Y12 inhibitors in platelet-mediated activation of neutrophils and monocytes. Thromb Haemost 114: 478-489, 2015.

12. Muzaffar S, Shukla N, Massey Y, Angelini GD and Jeremy JY: NADPH oxidase 1 mediates upregulation of thromboxane A2 synthase in human vascular smooth muscle cells: Inhibition with iloprost. Eur J Pharmacol 658: 187-192, 2011.

13. Henderson JT, Whitlock EP, O'Connor E, Senger CA, Thompson JH and Rowland MG: Low-dose aspirin for prevention of morbidity and mortality from preeclampsia: A systematic evidence review for the U.S. Preventive Services Task Force. Ann Intern Med 160: 695-703, 2014.

14. Rey E and Rivard GE: Is testing for aspirin response worthwhile in high-risk pregnancy? Eur J Obstet Gynecol Reprod Biol 157: 38-42, 2011.

15. National Research Council; Division on Earth and Life Studies Institute for Laboratory Animal Research; Committee for the Update of the Guide for the Care and Use of Laboratory Animals Washington, D.C: National Academies Press, Guide for the Care and Use of Laboratory Animals: Eighth edition-Thai version, 2011.

16. Chaiworapongsa T, Chaemsaithong P, Yeo L and Romero R: Pre-eclampsia part 1: Current understanding of its pathophysiology. Nat Rev Nephrol 10: 466-480, 2014.

17. Wallis AB, Saftlas AF and Hsia J: Secular trends in the rates of preeclampsia, eclampsia, and gestational hypertension, United States, 1987-2004. Am J Hypertens 21: 521-526, 2008

18. Bussolino F, Benedetto C and Massobrio M: Maternal vascular prostacyclin activity in pre-eclampsia. Lancet 2: 702, 1980.

19. Broughton Pipkin F, Crowther C, de Swiet M, Duley L, Judd A, Lilford RJ, Onwude J, Prentice C, Redman CW, Roberts J, et al: Where next for prophylaxis against pre-eclampsia? $\mathrm{Br} \mathrm{J}$ Obstet Gynaecol 103: 603-607, 1996.

20. Burton GJ and Jauniaux E: Oxidative stress. Best Pract Res Clin Obstet Gynaecol 25: 287-299, 2011.

21. Mikhail MS, Anyaegbunam A and Garfinkel D: Preeclampsia and antioxidant nutrients: decreased plasma levels of reduced ascorbic acid, alpha-tocopherol, and beta-carotene in women with preeclampsia. Am J Obstet Gynecol 171: 150-157, 1994

22. Rocha S, Gomes D, Lima M, Bronze-da-Rocha E and Santos-Silva A: Peroxiredoxin 2, glutathione peroxidase, and catalase in the cytosol and membrane of erythrocytes under $\mathrm{H}_{2} \mathrm{O}_{2}$-induced oxidative stress. Free Radic Res 49: 990-1003, 2015 .
23. Putnam CD, Arvai AS, Bourne Y and Tainer JA: Active and inhibited human catalase structures: Ligand and NADPH binding and catalytic mechanism. J Mol Biol 296: 295-309, 2000

24. Mahmood DF, Abderrazak A,El Hadri K, Simmet T and Rouis M: The thioredoxin system as a therapeutic target in human health and disease. Antioxid Redox Signal 19: 1266-1303, 2013.

25. Rhee SG, Chae HZ and Kim K: Peroxiredoxins: A historical overview and speculative preview of novel mechanisms and emerging concepts in cell signaling. Free Radic Biol Med 38 1543-1552, 2005

26. Mustacich D and Powis G: Thioredoxin reductase. Biochem J 346: 1-8, 2000

27. Rhee SG, Yang KS, Kang SW, Woo HA and Chang TS: Controlled elimination of intracellular $\mathrm{H}(2) \mathrm{O}(2)$ : Regulation of peroxiredoxin, catalase, and glutathione peroxidase via post-translational modification. Antioxid Redox Signal 7: 619-626, .2005.

28. Fischer LR, Igoudjil A, Magrané J, Li Y, Hansen JM, Manfredi G and Glass JD: SOD1 targeted to the mitochondrial intermembrane space prevents motor neuropathy in the Sod1 knockout mouse. Brain 134: 196-209, 2011.

29. Pizzino G, Irrera N, Cucinotta M, Pallio G, Mannino F, Arcoraci V, Squadrito F, Altavilla D and Bitto A: Oxidative stress: Harms and benefits for human health. Oxid Med Cell Longev 2017: 8416763, 2017.

30. Travaglino A, Raffone A, Saccone G, Migliorini S, Maruotti GM, Esposito G, Mollo A, Martinelli P, Zullo F and D'Armiento M: Placental morphology, apoptosis, angiogenesis and epithelial mechanisms in early-onset preeclampsia. Eur J Obstet Gynecol Reprod Biol 234: 200-206, 2019.

31. Cairns RA, Harris IS and Mak TW: Regulation of cancer cell metabolism. Nat Rev Cancer 11: 85-95, 2011.

32. Keum D, Kruse M,Kim DI, Hille B and Suh BC: Phosphoinositide 5- and 3-phosphatase activities of a voltage-sensing phosphatase in living cells show identical voltage dependence. Proc Natl Acad Sci USA 113: E3686-E3695, 2016.

33. Fruman DA, Chiu H, Hopkins BD, Bagrodia S, Cantley LC and Abraham RT: The PI3K pathway in human disease. Cell 170: 605-635, 2017.

34. Li C, Li Y, He L, Agarwal AR, Zeng N, Cadenas E and Stiles BL: PI3K/AKT signaling regulates bioenergetics in immortalized hepatocytes. Free Radic Biol Med 60: 29-40, 2013.

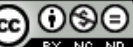

This work is licensed under a Creative Commons Attribution-NonCommercial-NoDerivatives 4.0 International (CC BY-NC-ND 4.0) License. 